\title{
Índices de qualidade e crescimento de mudas de café produzidas em tubetes
}

\author{
Seedling quality in coffee grown in containers
}

\author{
João Paulo Marana ${ }^{\mathrm{I}}$ Édison Miglioranza ${ }^{\mathrm{II}}$ Ésio de Pádua Fonseca $^{\mathrm{II}}$ \\ Roberto Hiroshi KainumaIII
}

\section{RESUMO}

O objetivo deste trabalho foi estudar os efeitos das doses de fertilizante de liberação lenta e dois substratos sobre o crescimento e a qualidade das mudas de Coffea arabica L., a fim de gerar parâmetros que permitam a padronização e a classificação da qualidade de cada lote de mudas. Foram utilizados dois substratos (substrato comercial Plantmax ${ }^{\circledast}$ e o vermicomposto de esterco de curral com casca de arroz carbonizada na proporção de 4:1 v/v) e cinco doses de fertilizante de liberação lenta: $0,5,10,15,20 \mathrm{~kg} \mathrm{~m}^{-3}$ de substrato em tubetes com plântulas de Catuaí Vermelho IAC 99, conduzidas em viveiro coberto por tela de polipropileno com $50 \%$ de sombreamento. Cinco meses após emergência, foram determinados a matéria seca de raiz, caule, folha, a matéria seca total, o número de folhas, a área foliar, o diâmetro de caule, a altura da parte aérea, a relação parte aérea raiz, a relação altura da planta e diâmetro do coleto e o índice de qualidade de Dickson. Para cada característica avaliada dentro de cada substrato, foram testados modelos polinomiais para o efeito de doses de adubo de liberação lenta. Os substratos necessitaram de adubação para garantir o crescimento $e$ desenvolvimento adequado das mudas. A dose de $10 \mathrm{~kg}$ de fertilizante de liberação lenta por $\mathrm{m}^{3}$ de substrato foi a mais adequada, independentemente do substrato utilizado. Nessa dose, o substrato comercial apresentou desempenho superior para todos os parâmetros de crescimento avaliados. As mudas desenvolvidas em substrato comercial adubado com $10 \mathrm{~kg}$ de fertilizante de liberação lenta por $\mathrm{m}^{-3}$ apresentaram a relação altura da planta e o diâmetro do caule de 4,0; o índice de qualidade de Dickson de 0,21 e a relação de matéria seca da parte aérea e da raiz de 4,7.

Palavras-chave: Coffea arabica L., propagação, adubação, qualidade fisiológica, índice de Dickson.

\begin{abstract}
This experiment was designed to evaluate the effect of slow-releasing fertilizer and substrates on the growth of coffee seedlings, in order to generate parameters to allow classification and the standardization of the seedling quality. Seeds from Coffea arabica L. cv. Catuaí Vermelho IAC 99 were sowing in plastic containners (120mL volume) and conducte in a nursery covered with polipropileno screen with $50 \%$ shade. The experimental design consisted of factorial randomized blocks: two substrates (a commercial and vermicompost of cow manure and carbonized rice hull in 4:1 v/v proportion) $x 5$ doses of slow-releasing fertilizer $\left(0,5,10,15\right.$ and $20 \mathrm{~kg} \mathrm{~m}^{-3}$ of substrate). The evaluation was carried out five months after emergence. The evaluated parameters were: root dry mass, stem dry mass, leaf dry mass, total dry mass, number of leaves, foliar area, stem diameter, shoot height, shoot/root ratio, plant height/ stem diameter ratio and the Dickson quality index. Both substrates needed fertilizer to produce seedlings with minimal commercial quality. The recommended dosage of slow-releasing fertilizer for coffee seedlings production was $10 \mathrm{~kg} . \mathrm{m}^{-3}$. The seedlings that grew in slow-releasing fertilizer $\left(10 \mathrm{~kg} . \mathrm{m}^{-3}\right)$ fertilized Plantmax ${ }^{\circledast}$ presented: plant height/stem diameter ratio equal 4:0; Dickson quality index 2.10, and the shoot dry mass/ root dry mass ratio, equal 4.7 .
\end{abstract}

Key words: Coffea arabica L., nursery, fertilizer, Dickson index.

\section{INTRODUÇÃO}

A produção de café no Brasil tem importância tanto econômica como social, pois o país é o maior produtor mundial, e a cultura utiliza predominante mãode-obra da produção de mudas até a colheita.

IPrograma de Pós-graduação em Agronomia, Universidade Estadual de Londrina (UEL), Londrina, PR, Brasil.

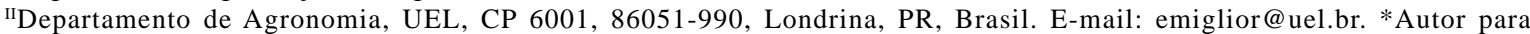
correspondência.

IIICurso de Agronomia, UEL, Londrina, PR, Brasil. 
Na produção comercial de mudas de café tradicionalmente são empregadas sacolas de polietileno. Esses recipientes, porém, trazem o inconveniente de necessitarem de maior volume de substrato, o que aumenta a área do viveiro e dificulta o manejo, especialmente com relação à distribuição de água e mondas e, posteriormente, o transporte para o campo e a posterior distribuição para o plantio na lavoura (CAMPINHOS JR. \& IKEMORI, 1983). Além disso, maior quantidade de substrato amplia a possibilidade de disseminação de pragas e doenças, o que pode inviabilizar futuros cultivos.

Por outro lado, o uso de tubetes constituise numa alternativa para solucionar esses problemas com vantagens (SIMÕES, 1987). Tais recipientes já vêm sendo utilizados com sucesso, por um bom período, em viveiros de pínus e de eucaliptos, com redução nos custos de produção de aproximadamente $50 \%$ para mudas de eucaliptos (CAMPINHOS JR \& IKEMORI, 1983). A produção de mudas de café em tubetes vem sendo efetuada desde 1989 e atualmente é usada em quase todo o Brasil (COSTA et al., 2000).

No caso da utilização de tubetes na produção de mudas, não são recomendados substratos com predominância de terra ou areia (GOMES et al., 1985). Por sua vez, a casca de arroz carbonizada, devido às suas características físicas, químicas e biológicas, é potencialmente utilizável (MINAMI \& GONÇALVES, 1994).

O vermicomposto, ou húmus de minhoca, é outro substrato promissor e vem sendo estudado especialmente na produção de mudas de espécies florestais (SCHUMACHER et al., 2001; VOGEL et al., 2001), sendo usado também como substrato na produção de mudas de café (ANDRADE NETO et al., 1999).

O substrato comercial Plantmax ${ }^{\circledR}$, devido às suas boas características físicas, também pode ser utilizado na produção de mudas de café, embora necessite de adubações complementares. Na verdade, devido ao pequeno volume dos tubetes, os substratos normalmente utilizados em seu preenchimento não conseguem fornecer as quantidades de nutrientes adequados para o crescimento e desenvolvimento das mudas (MÜLLER et al., 1997; ANDRADE NETO et al., 1999; COSTA et al., 2000). Dessa forma, os nutrientes devem ser fornecidos na forma sólida ou por meio de fertirrigação. MÜLLER et al. (1997) conseguiram produzir mudas de café de boa qualidade em tubetes com o uso de mistura de vermiculita e casca carbonizada de arroz, acrescidas de doses adequadas de nitrogênio, fósforo e potássio, fornecidas via irrigação. Entretanto, os viveiristas produtores de mudas de café geralmente não possuem estrutura suficiente para realizar a fertirrigação em larga escala, enquanto os adubos sólidos tradicionais, devido ao volume limitado dos tubetes, não podem ser fornecidos de uma só vez, por ocasião da semeadura ou mesmo do transplantio.

Os nutrientes a serem fornecidos às mudas devem ser disponibilizados de acordo com a necessidade das mesmas, durante o período de cinco meses que é necessário à sua formação. O uso de um adubo com liberação lenta atende a essa questão, podendo assim servir para a produção de mudas de café em tubetes, com qualidade necessária para a comercialização.

Nesse sistema de produção, a avaliação da qualidade da muda pode ser uma ferramenta para identificar se está sendo conduzida de maneira adequada, isto é, se as mudas encontram-se sadias, com o máximo potencial para sobrevivência e posterior desenvolvimento no campo (FONSECA, 2000).

O princípio de avaliação quantitativa é de que quanto maior a muda melhor. Mas, para evitar distorções provenientes do excesso de nitrogênio, por exemplo, ou do crescimento foliar em detrimento do sistema radicular, utilizam-se índices de qualidade, que são relações entre os parâmetros de crescimento.

Neste trabalho, o objetivo foi estudar os efeitos das doses de adubo de liberação lenta e de dois tipos de substratos sobre as diversas características de crescimento e sobre a qualidade das mudas de café, a fim de obterem-se parâmetros que permitam a padronização e a classificação segura da qualidade de um determinado lote de mudas.

\section{MATERIAL E MÉTODOS}

O experimento foi desenvolvido no período de fevereiro a julho de 2001, no setor de produção de mudas, no Centro de Ciências Agrárias, na Universidade Estadual de Londrina, em Londrina Paraná, com latitude $23^{\circ} 23^{\prime} \mathrm{S}$, longitude $51^{\circ} 11^{\prime} \mathrm{W}$. O clima da região é Cfa, segundo a classificação de W. Köppen.

Foram utilizados dois substratos (substrato comercial Plantmax ${ }^{\circledR}$ e o vermicomposto de esterco de curral com casca de arroz carbonizada na proporção de 4:1 v/v) e cinco doses de fertilizante de liberação lenta: $0,5,10,15,20 \mathrm{~kg} \mathrm{~m}^{-3}$ de substrato. O substrato Plantmax ${ }^{\circledR}$ e o substrato vermicomposto apresentavam respectivamente: nitrogênio 5,5 $\mathrm{cmol}_{c} \mathrm{dm}^{-3}$ e 5,5 $\mathrm{cmol}_{c}$ $\mathrm{dm}^{-3}$; fósforo $2,5 \mathrm{cmol} \mathrm{dm}^{-3} \mathrm{e} 194,3 \mathrm{cmol} \mathrm{dm}^{-3}$; potássio 4,6 $\mathrm{cmol} \mathrm{dm}^{-3}$ e 4,4 $\mathrm{cmol} \mathrm{dm}^{-3}$; cálcio $15,5 \mathrm{cmol} \mathrm{dm}^{-3} \mathrm{e}$ $9,9 \mathrm{cmol}_{\mathrm{c}} \mathrm{dm}^{-3}$; magnésio $24,7 \mathrm{cmol}_{\mathrm{c}} \mathrm{dm}^{-3}$ e $8,7 \mathrm{cmol}_{\mathrm{c}} \mathrm{dm}^{-3}$.

Ciência Rural, v.38, n.1, jan-fev, 2008. 
O adubo de liberação lenta utilizado foi o Osmocote ${ }^{\circledR}$, com a seguinte composição química: $\mathrm{N}=15 \%$, (7\% amoniacal e $8 \%$ nitrato), $\mathrm{P}_{2} \mathrm{O}_{5}=9 \%, \mathrm{~K}_{2} \mathrm{O}=12 \%, \mathrm{Ca}=$ $3,5 \%, \mathrm{~S}=2,3 \%, \mathrm{Mg}=1 \%, \mathrm{Fe}=0,45 \%, \mathrm{Mn}=0,06 \%, \mathrm{Cu}$ $=0,05 \%, \mathrm{Zn}=0,05 \%$ e $\mathrm{Mo}=0,02 \%$.

Os tubetes utilizados possuíam diâmetro na parte superior de $35 \mathrm{~mm}, 140 \mathrm{~mm}$ de altura e $120 \mathrm{~mL}$ de capacidade volumétrica. Todos os tubetes foram previamente lavados e esterilizados com hipoclorito de sódio a $2 \%$ diluído em água. Foram utilizadas duas sementes de café, da cultivar "IAC 99", por tubete, sendo estes mantidos no setor de semeadura e germinação, que é protegido com plástico transparente e tela de polietileno de coloração preta, com retenção de $48 \%$ do fluxo da radiação solar, e provido de sistema de irrigação suspenso com microaspersores com vazão de $75 \mathrm{l} \mathrm{h}^{-1}$. Posteriormente à germinação, as mudas foram levadas para o viveiro coberto com sombrite com $50 \%$ de sombreamento e desbastadas, deixando-se apenas uma plântula por tubete. Rega, monda, controle de pragas e doenças foram efetuados conforme recomendação para mudas de café.

O delineamento experimental foi o de blocos casualizados em esquema fatorial, com dois substratos e cinco doses de adubo de liberação lenta, com quatro repetições e 25 plantas por parcela, distribuídas em cinco linhas e cinco colunas.

A avaliação do crescimento e da qualidade da muda ocorreu aos 150 dias após a semeadura (DAS). Foram determinadas as seguintes características: a) área foliar, expressa em $\mathrm{cm}^{2}$, estimada com medidor de área foliar LI-COR modelo LI-3000; b) número de folhas; c) altura da parte aérea, expressa em cm, medida com régua milimetrada, a partir do coleto até a gema apical; d) diâmetro do coleto, expresso em mm, medido utilizandose um paquímetro com precisão de $0,01 \mathrm{~mm}$; e) matéria seca de folhas, matéria seca do caule e matéria seca de raízes, expressas em gramas, determinadas em estufa de circulação forçada a $75^{\circ} \mathrm{C}$ até peso constante; f) matéria seca total, expressa em gramas, obtida pela soma das matérias secas de folhas caule e raiz; g) RPAR: relação da matéria seca da parte aérea com a matéria seca de raízes; h) RAD: relação da altura parte aérea com o diâmetro do coleto; i) IQD: índice de qualidade de Dickson obtido pela fórmula j) IQD = [matéria seca total/(RAD + RPAR)] (DICKSON et al., 1960).

Para cada característica avaliada dentro de cada substrato, foram testados modelos polinomiais para o efeito de doses de adubo de liberação lenta. O critério para a escolha do modelo foi a significância pelo teste $\mathrm{F}$ a $5 \%$ de probabilidade de erro que tenha apresentado maior valor de coeficiente de determinação $\left(\mathrm{r}^{2}\right)$. Os pontos de máximo foram determinados por meio das derivada primeira das equações de regressão.

\section{RESULTADOS E DISCUSSÃO}

O resultado da análise de variância mostrou efeito significativo para doses e para substrato. Após a realização do teste $\mathrm{F}$ para determinar o grau das equações, obteve-se que o efeito das doses de adubo de liberação lenta sobre todas as variáveis foi positivo quadrático para o substrato Plantmax-café ${ }^{\circledR}$, assim como para o substrato vermicomposto, excetuando-se a área foliar (AF) e o diâmetro do coleto (DC), cujos efeitos foram positivos quadráticos (Figura 1).

Analisando o comportamento das funções da figura 1, para o substrato vermicomposto, observase que foram alcançados pontos de máximo crescimento correspondentes às doses de adubo de liberação lenta, que variaram de $13,75 \mathrm{~kg} \mathrm{~m}^{-3}$ para diâmetro de coleto (DC) até $18,42 \mathrm{~kg} \mathrm{~m}^{-3}$ para relação altura da parte aérea/ diâmetro do coleto (RAD). Para o substrato Plantmaxcafé ${ }^{\circledR}$, os pontos de máximo crescimento variaram de $13,31 \mathrm{~kg} \mathrm{~m}^{-3}$ para o diâmetro de coleto (DC) até $15,28 \mathrm{~kg}$ $\mathrm{m}^{-3}$ para matéria seca de folhas (MSF).

De acordo com os gráficos da figura 1 , observa-se que, na dose zero de adubo de liberação lenta, as características de crescimento das mudas estudadas apresentaram os menores valores. Isso comprova que existe a necessidade de complementação nutricional para um bom desenvolvimento das mudas em tubetes, independentemente do substrato, como também foi encontrado por MÜLLER et al. (1997). Na dose zero de adubo de liberação lenta, o desenvolvimento das mudas foi melhor no substrato vermicomposto, para todas as características de crescimento avaliadas, o que pode ser explicado pela fertilidade inicial do substrato principalmente em relação ao fósforo.

Por outro lado, a dose de $5 \mathrm{~kg} \mathrm{~m}^{-3}$ de adubo de liberação lenta foi suficiente para o substrato Plantmax-café $^{\circledR}$ ser superior ao substrato vermicomposto nas características avaliadas, exceto para diâmetro do coleto (DC), mas ainda insuficiente para que a muda de café desenvolvesse o máximo de sua potencialidade (Figura 1).

É interessante notar que, na dose de $10 \mathrm{~kg} \mathrm{~m}^{-3}$, todas as características de crescimento avaliadas apresentaram melhor desempenho quando o substrato era o Plantmax-cafée ${ }^{\circledR}$. Isso inverte o que aconteceu com a dose zero de adubo de liberação lenta, na qual o substrato vermicomposto apresentou melhores resultados. Por outro lado, na dose de $15 \mathrm{~kg} \mathrm{~m}^{-3}$, o Plantmax-café ${ }^{\circledast}$ também apresentou comportamento superior, enquanto que, para a dose de $20 \mathrm{~kg} \mathrm{~m}^{-3}$, as características diâmetro do coleto (DC) e a relação altura 


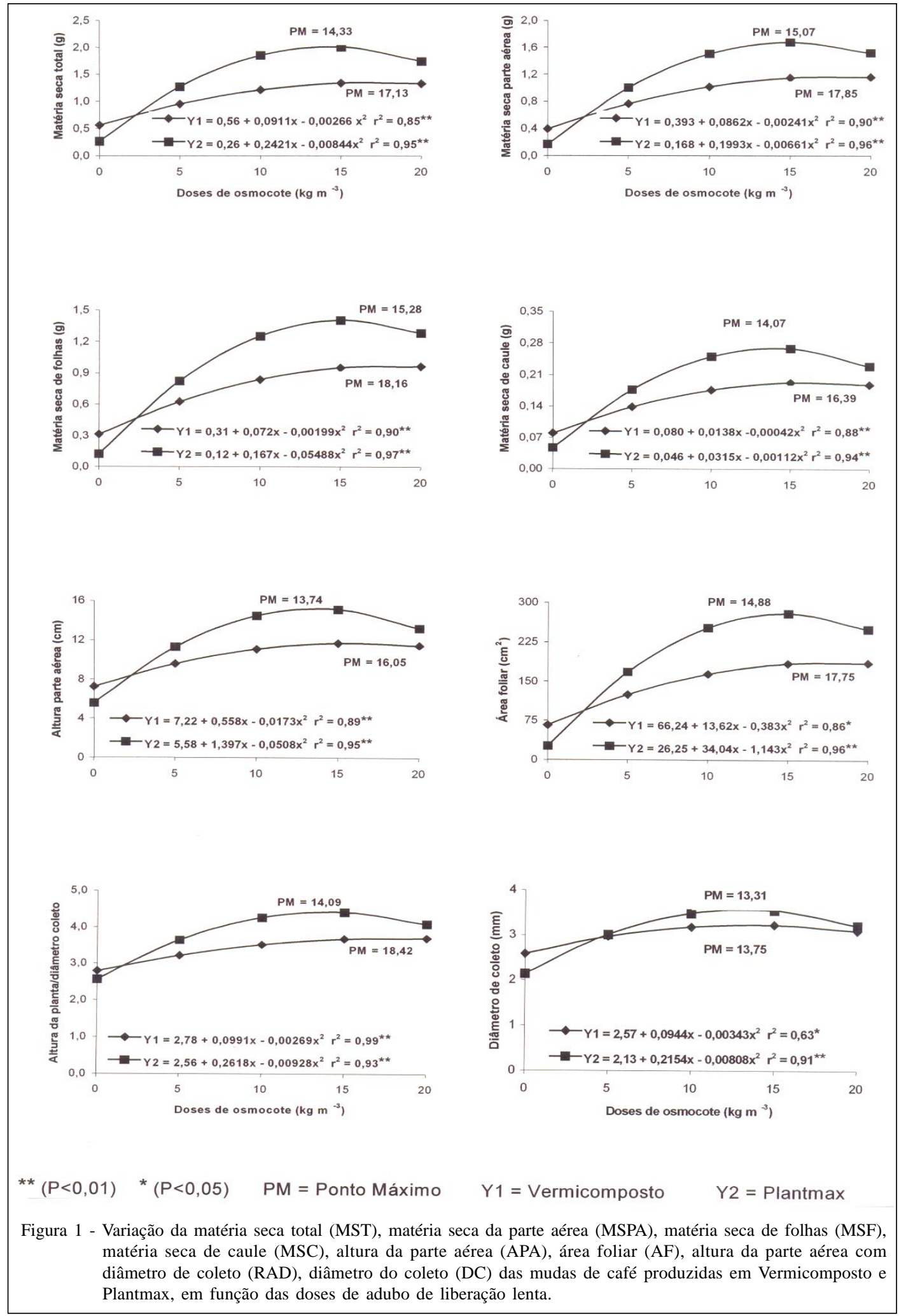

da parte aérea/ diâmetro do coleto (RAD) foram praticamente iguais em relação aos dois substratos (Figura 1).
O substrato Plantmax-café ${ }^{\oplus}$, fertilizado com adubo de liberação lenta, foi considerado adequado para a produção de mudas de café segundo ANDRADE

Ciência Rural, v.38, n.1, jan-fev, 2008. 
NETO et al. (1999), apesar de naquele experimento o Plantmax $^{\circledR}$ apresentar comportamento inferior ao esterco de curral em dosagem acima de 50\%. Estercos com maior concentração de nutrientes, como base do substrato, mesmo que curtido, podem não promover um adequado desenvolvimento das mudas, dependendo da forma como for usado (SILVA Jr. \& GIORGI, 1993).

As causas do melhor desempenho do Plantmax-café ${ }^{\circledR}$ que o do vermicomposto (Figura 1) devem ser explicadas pelas diferenças físicas e químicas entre os dois substratos. Uma possível causa é a de que a casca de arroz carbonizada, usada na composição do vermicomposto, possa ter contribuído para aumentar o teor de manganês, sendo que este, somado ao Mn acrescentado pelo adubo de liberação lenta, pode ter reduzido o crescimento das mudas. FRANCO et al. (2004) determinaram que a casca de arroz carbonizada apresenta altos teores de $\mathrm{Mn}$, cerca de $705 \mathrm{mg} \mathrm{kg}^{-1}$ de matéria seca.

Na tabela 1, estão apresentados os valores médios do que se consideraram índices de qualidade das mudas. Outras características, que não o RPAR, apresentaram interação significativa entre os substratos e doses de adubo de liberação lenta em nível de 5\% de probabilidade, pelo teste F.

Uma vez que os parâmetros de desempenho foram calculados a partir das características de crescimento, foram observados também os menores valores quando da ausência de adubação, independentemente do substrato utilizado, exceto para matéria seca de raiz no substrato vermicomposto (Tabela 1).

Os menores valores de matéria seca total (MST) foram encontrados na dose zero de adubação, quando se comparam as doses de um mesmo substrato.

Tabela 1 - Parâmetros de crescimento e qualidade de mudas de café: relação parte aérea raiz (RPAR), índice de qualidade de Dickson (IQD) e relação altura da planta e diâmetro do coleto (RAD), em função dos tipos de substratos com cinco diferentes doses de adubo de liberação lenta.

\begin{tabular}{|c|c|c|c|c|c|c|c|c|}
\hline \multirow{2}{*}{$\begin{array}{l}\text { Parâmetros de } \\
\text { crescimento }\end{array}$} & \multirow[b]{2}{*}{ Substrato } & \multicolumn{7}{|c|}{ 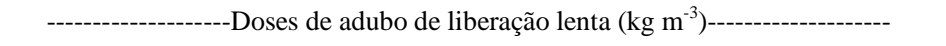 } \\
\hline & & 0 & 5 & 10 & & 15 & & 20 \\
\hline \multirow{2}{*}{$\begin{array}{l}\text { Massa seca } \\
\text { raiz (g) }\end{array}$} & Vermicomposto & 0,15 a & $0,23 \mathrm{a}$ & $0,16 \mathrm{~b}$ & \multicolumn{2}{|c|}{$0,17 \mathrm{~b}$} & $0,17 \mathrm{a}$ & \\
\hline & Plantmax & $0,07 \mathrm{~b}$ & $0,31 \mathrm{a}$ & 0,33 a & \multicolumn{2}{|c|}{$0,29 \mathrm{a}$} & $0,24 \mathrm{a}$ & \\
\hline Massa seca & Vermicomposto & 0,06 a & $0,16 \mathrm{~b}$ & $0,17 \mathrm{~b}$ & \multicolumn{2}{|c|}{$0,17 \mathrm{~b}$} & 0,17 a & \\
\hline caule (g) & Plantmax & $0,03 \mathrm{~b}$ & 0,20 a & 0,24 a & \multicolumn{2}{|c|}{$0,24 \mathrm{a}$} & $0,24 \mathrm{a}$ & \\
\hline Massa seca & Vermicomposto & 0,25 a & $0,76 \mathrm{~b}$ & $0,80 \mathrm{~b}$ & \multicolumn{2}{|c|}{$0,86 \mathrm{~b}$} & $1,01 \mathrm{~b}$ & \\
\hline folhas (g) & Plantmax & $0,05 \mathrm{~b}$ & 0,97 a & $1,20 \mathrm{a}$ & \multicolumn{2}{|c|}{$1,31 \mathrm{a}$} & 1,33 a & \\
\hline \multirow{2}{*}{ Massa seca total (g) } & Vermicomposto & 0,47 a & $1,15 \mathrm{~b}$ & $1,13 \mathrm{~b}$ & \multicolumn{2}{|c|}{$1,21 \mathrm{~b}$} & $1,38 \mathrm{~b}$ & \\
\hline & Plantmax & $0,16 b$ & 1,49 a & $1,77 \mathrm{a}$ & \multicolumn{2}{|c|}{1,86 a } & $1,57 \mathrm{a}$ & \\
\hline \multirow{2}{*}{$\begin{array}{l}\text { Número de } \\
\text { folhas }\end{array}$} & Vermicomposto & 7,31 a & $10,43 \mathrm{a}$ & $9,87 \mathrm{~b}$ & \multicolumn{2}{|c|}{$10,43 \mathrm{~b}$} & $10,25 \mathrm{~b}$ & \\
\hline & Plantmax & $3,68 \mathrm{~b}$ & $10,62 \mathrm{a}$ & $11,5 \mathrm{a}$ & \multicolumn{2}{|c|}{11,81 a } & 11,37 a & \\
\hline \multirow{2}{*}{ Área foliar $\left(\mathrm{cm}^{2}\right)$} & Vermicomposto & $52,81 \mathrm{a}$ & 156,67 b & $149,08 \mathrm{~b}$ & \multicolumn{2}{|c|}{$172,54 \mathrm{~b}$} & \multicolumn{2}{|c|}{$193,72 \mathrm{~b}$} \\
\hline & Plantmax & $12,36 \mathrm{~b}$ & 199,80 a & 239,90 a & \multicolumn{2}{|c|}{264,22 a } & \multicolumn{2}{|c|}{259,39 a } \\
\hline \multirow{2}{*}{$\begin{array}{l}\text { Diâmetro de } \\
\text { coleto (mm) }\end{array}$} & Vermicomposto & $2,44 \mathrm{a}$ & 3,27 a & $3,06 \mathrm{~b}$ & \multicolumn{2}{|c|}{3,06 a } & \multicolumn{2}{|l|}{3,19 a } \\
\hline & Plantmax & 2,02 b & 3,23 a & 3,48 a & \multicolumn{2}{|c|}{3,31 a } & \multicolumn{2}{|l|}{3,32 a } \\
\hline \multirow{2}{*}{$\begin{array}{l}\text { Altura da parte } \\
\text { aérea }(\mathrm{cm})\end{array}$} & Vermicomposto & 6,79 a & $10,62 \mathrm{~b}$ & $10,57 \mathrm{~b}$ & \multicolumn{2}{|c|}{$11,31 \mathrm{~b}$} & \multicolumn{2}{|l|}{$11,70 \mathrm{~b}$} \\
\hline & Plantmax & $4,46 \mathrm{~b}$ & $12,72 \mathrm{a}$ & 13,85 a & \multicolumn{2}{|c|}{$14,50 \mathrm{a}$} & $13,60 \mathrm{a}$ & \\
\hline \multirow{2}{*}{$\begin{array}{l}\text { Parâmetro de } \\
\text { qualidade }\end{array}$} & Substrato & ----- & -----Dos & adubo de libe & ção lent & $\left.1^{-3}\right)------$ & 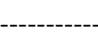 & \\
\hline & sudstrato & 0 & 5 & 10 & 15 & 20 & & média \\
\hline RAD & Vermicomposto & $2,77 \mathrm{a}$ & $3,26 b$ & $3,45 \mathrm{~b}$ & $3,69 \mathrm{~b}$ & $3,69 \mathrm{~b}$ & & ------ \\
\hline & Plantmax & $2,45 b$ & 3,94 a & $4,01 \mathrm{a}$ & $4,42 \mathrm{a}$ & $4,11 \mathrm{a}$ & & ------ \\
\hline IQD & Vermicomposto & 0,09 a & 0,15 a & $0,11 \mathrm{~b}$ & $0,12 \mathrm{~b}$ & 0,13 a & & ------ \\
\hline & Plantmax & $0,04 \mathrm{~b}$ & 0,19 a & $0,21 \mathrm{a}$ & 0,19 a & 0,16 a & & ----- \\
\hline RPAR & Vermicomposto & 2,07 & 4,40 & 6,95 & 6,56 & 7,95 & & 5,59 a \\
\hline & Plantmax & 1,14 & 4,27 & 4,71 & 6,06 & 7,56 & & $4,75 \mathrm{~b}$ \\
\hline & Média & 1,60 & 4,33 & 5,83 & 6,31 & 7,75 & & \\
\hline
\end{tabular}

Médias não precedidas de mesma letra na vertical (entre substratos) diferem entre si em nível de 1\% pelo Teste de Tukey.

Ciência Rural, v.38, n.1, jan-fev, 2008. 
No entanto, quando, na dose zero, comparam-se os dois substratos, a MST foi maior para o vermicomposto que para o Plantmax-café ${ }^{\circledast}$. Entretanto, já a partir da dose $5 \mathrm{~kg} \mathrm{~m}^{-3}$, o Plantmax-café ${ }^{\circledast}$ apresentou melhor desempenho do que o vermicomposto, comprovando sua alta eficiência como adubo de liberação lenta para suplementação de nutrientes em substrato para o crescimento de mudas de café (ANDRADE NETO et al., 1999). Os valores de MST variaram entre 0,16 e 1,86. Considerando-se que a dose de $10 \mathrm{~kg}$ de adubo de liberação lenta por $\mathrm{m}^{3}$ de substrato foi a mais adequada para o crescimento e desenvolvimento do cafeeiro (Tabela 1), valores de MST entre 1,0 e 1,8 gramas de matéria seca por muda parecem razoáveis.

As relações altura da planta/diâmetro do coleto (RAD) também foram menores na dose zero de adubação e, novamente, as plantas se desenvolveram melhor em Vermicomposto que em Plantmax ${ }^{\circledR}$ (Tabela 1). Em todas as adubações com adubo de liberação lenta, as mudas também apresentaram melhor desempenho quando o substrato usado foi o Plantmax ${ }^{\circledR}$. No caso do vermicomposto, os valores de RAD variaram entre 2,77 e 3,69, e os maiores valores foram obtidos para as doses de 10, 15 e $20 \mathrm{~kg} \mathrm{~m}^{-3}$ (Tabela 1). Com relação ao Plantmax ${ }^{\circledR}$, os valores de RAD variaram entre 2,45 e 4,42, e os maiores valores foram obtidos para as doses de 15 e $20 \mathrm{~kg} \mathrm{~m}^{-3}$ (Tabela 1 ).

De maneira geral, observou-se que, com o incremento das doses de adubo, aumentaram os valores de RAD, indicando um efeito semelhante ao estiolamento, crescendo a muda mais em altura do que em diâmetro do coleto. Considerando-se que a dose de $10 \mathrm{~kg} \mathrm{~m}^{-3}$ foi a mais adequada, então, valores de RAD de 3,5 a 4,0 parecem razoáveis. Valores maiores indicam crescimento excessivo da muda em altura, e menores indicam menor crescimento. Em ambos os casos, os problemas podem ser controlados com o manejo das condições no viveiro. O crescimento excessivo pode ser controlado com reduções nas adubações, reduções na irrigação e exposição à plena luz.

Os índices de qualidade de Dickson variaram entre 0,04 e 0,21 (Tabela 1). Quando o substrato foi o vermicomposto, a dose zero apresentou a menor média e a dose de $5 \mathrm{~kg} \mathrm{~m}^{-3}$ apresentou a maior média. Por outro lado, nas demais doses, os desempenhos das mudas foram intermediários entre esses dois extremos (Tabela 1). No caso do Plantmax ${ }^{\circledR}$, o menor valor foi obtido na dose zero, sendo que, para todas as demais doses, as médias foram maiores que essa. No tratamento sem adubação, o vermicomposto apresentou melhores resultados que o Plantmax-café ${ }^{\circledR}$. Estabelecendo-se como padrão o valor mínimo de 0,20, conforme recomendação de HUNT (1990), observa-se que apenas na dose de $10 \mathrm{~kg}$ de adubo de liberação lenta por $\mathrm{m}^{3} \mathrm{de}$ Plantmax $^{\circledast}$ as mudas atingiram esse valor. Conforme FONSECA (2000), o manejo das mudas no viveiro pode permitir que se atinja o valor mínimo desejado.

Os valores da relação parte aérea/raiz (RPAR) variaram entre 1,14 e 7,95 (Tabela 1). De modo geral, RPAR aumentou com o aumento da dose de adubo fornecido, independentemente do tipo de substrato. Assim, somente com a dosagem máxima obteve-se a maior média entre os dois substratos para a relação parte aérea/raiz (RPAR), que foi de 7,75 (Tabela 1). As mudas produzidas no substrato Plantmax ${ }^{\circledR}$ apresentaram média menor que as produzidas no substrato vermicomposto, evidenciando que as mudas de café apresentam maior crescimento de suas raízes quando desenvolvidas no vermicomposto, em relação ao Plantmax ${ }^{\circledR}$. Considerando-se a dose de adubo de liberação lenta de $10 \mathrm{~kg} \mathrm{~m}^{-3}$ de substrato como a mais adequada para o desenvolvimento das mudas, valores razoáveis de RPAR podem variar de 4,7 a 7,0. Valores de RPAR menores que 4,7 indicam que a muda não teve um bom desenvolvimento da parte aérea, sendo que, acima de 7,0, o crescimento do sistema radicular aparentemente foi insuficiente.

Os índices estudados relacionam-se com um equilíbrio entre diferentes avaliações, através de uma razão entre elas. Identificou-se, neste trabalho, para o RPAR, que os melhores valores situaram-se entre quatro e sete, o que é semelhante ao encontrado em MÜLLER et al. (1997), que não utilizaram adubo de liberação lenta em seu estudo, o que melhor caracteriza a validade de sua referência para uso generalizado.

\section{CONCLUSÕES}

Em tubetes de $120 \mathrm{~mL}$, os substratos necessitaram de adubação para garantir o crescimento e o desenvolvimento adequado das mudas de café, sendo a dose de $10 \mathrm{~kg}$ de adubo de liberação lenta por $\mathrm{m}^{3}$ de substrato a mais adequada para a formação de mudas de café, independentemente do substrato utilizado.

Quando se adubou com 10kg de adubo de liberação lenta por $\mathrm{m}^{3}$, no que se refere à qualidade de muda, foi preferível usar o Plantmax-café ${ }^{\circledR}$, em relação ao vermicomposto. As mudas desenvolvidas em Plantmax-café ${ }^{\circledR}$ adubado com $10 \mathrm{~kg}$ de adubo de liberação lenta por $\mathrm{m}^{3}$ apresentaram o valor de relação altura da planta e o diâmetro do caule de 4,0; o índice de qualidade de Dickson de 0,21 ; e a relação de matéria seca entre a parte aérea e raiz de 4,7. 


\section{REFERÊNCIAS}

ANDRADE NETO, A. et al. Avaliação de substratos alternativos e tipos de adubação para a produção de mudas de cafeeiro (Coffea arabica L.) em tubetes. Ciência e Agrotecnologia, v.23, n.2, p.270-280, 1999.

CAMPINHOS JÚNIOR, E.; IKEMORI, Y.K. Introdução de nova técnica na produção de mudas de essências florestais. Silvicultura, n.28, p.226-228, 1983.

COSTA, A.C.M. et al. Mudas em tubetes: novos componentes e misturas. Informativo da Cooperativa dos Cafeicultores da Região de Garça, Ano 5, n.51, p.14-15, 2000.

DICKSON, A. et al. Quality appraisal of white spruce and white pine seedling stock in nurseries. Forest Chronicle, v.36 p.10-13, 1960

FONSECA, E.P. Padrão de qualidade de mudas de Trema micantra (L.) Blume, Cedrela fissilis Vell. e Aspisdosperma polyneurom Müll. Arg. produzidas sob diferentes períodos de sombreamento. 2000. 113f. Tese (Doutorado em Agronomia - Produção Vegetal) - Universidade Estadual Paulista.

FRANCO, E. et al. High metal contents in coffee plant organs developed in tubets with different proportions of biosolid composts and carbonized rice hulls. Brazilian Archives of Biology and Technology, v.47, n.4, p.503-510, 2004

GOMES, J.M. et al. Uso de diferentes substratos na produção de mudas de Eucaliptos grandis em tubetes e em bandejas de isopor. Revista Árvore, v.9, n.1, p.58-86, 1985.
HUNT, G.A. Effect of styroblock design and cooper treatment on morphology of conifer seedlings. In: TARGET SEEDLING SYMPOSIUM, MEETING OF THE WESTERN FOREST NURSERY ASSOCIATIONS, GENERAL TECHNICHAL REPORT RM-200, 1990, Roseburg. Proceedings... Fort Collins: United States Departament of Agriculture, Forest Service, 1990. p.218-222.

MINAMI, K.; GONÇALVES, A.L. Efeito de substrato artificial no enraizamento de Calanchoe (Kalanchoe x blossfeldiana cV. Singapur, Crassulaceae). Scientia Agricola, v.51, n.2, p.151155, 1994

MÜLLER, M.L. et al. Produção de mudas de cafeeiro (Coffea arabica L.) cv. Mundo Novo em tubetes. Revista Unimar, v.19, n.3, p.777-786, 1997

SCHUMACHER, M.V. et al. Influência de vermicomposto na produção de mudas de Eucalyptus grandis Hill ex Maiden. Ciência Florestal, v.11, n.2, p.01-08, 2001.

SILVA JÚNIOR, A.A.; GIORGI, E. Substratos alternativos para a produção de mudas de tomateiro. Florianópolis: EPAGRI, 1993. 59p. (Boletim Técnico).

SIMÕES, J.W. Problemática de produção de mudas em essências florestais. Série Técnica IPEF, v.4, n.13, p.1-6, 1987.

VOGEL, H.L.M. et al. Utilização de vermicomposto no crescimento de mudas de Hovenia dulcis Thunberg. Ciência Florestal, v.11, n.1, p.21-27, 2001. 